\title{
Terceiro molar impactado em região de seio maxilar Relato de caso
}

\section{Upper third molar impacted in region sinus maxillary Case report}

\begin{abstract}
RESUMO
Introdução: Os terceiros molares comumente estão em posições ectópicas, geralmente devido à impacção, causada pela falta de espaço no respectivo arco. Geralmente são encontrados em achados radiográficos, mas podem estar relacionados a sintomas apresentados pelos pacientes, como edema e dor. O tratamento pode ser conserva5 dor ou cirúrgico e irá depender das condições clínicas e/ou dos sintomas relatados pelo paciente.

Objetivo: $\mathrm{O}$ objetivo do artigo é o relato de caso de remoção cirúrgica de um terceiro molar impactado em região íntima ao seio maxilar relacionado a queixa de dor crônica da paciente.

Relato de caso: Paciente, gênero feminino, 29 anos, leucoderma, compareceu com queixa principal de dor intensa e contínua, na região maxilar superior esquerda. Os exames de imagem demonstraram a presença de um elemento dentário, intimamente relacionado ao seio maxilar. Esse foi removido por meio de técnica cirúrgica conservadora.

Conclusão: $O$ tratamento de dentes impactados requer a avaliação por um profissional capacitado para tomar a melhor decisão acerca da conduta para cada caso, podendo ser um tratamento conservador ou cirúrgico.

Palavras chave: Terceiro molar. Dente impactado. Seio maxilar.
\end{abstract}

\section{ABSTRACT}

Introduction: The third molars are commonly in ectopic positions, usually due to the impaction caused by the lack of space in the respective arch. They are found are found in radiographic findings, but may be related to factors related to patients, such as edema and pain.

Objective: The objective of the article is related to the case of surgical removal of an impacted third molar in the region close to the maxillary sinus related to the patient's chronic pain complaint.

Case Report: Female patient, 29 years old, leucoderma, attended the main complaint of severe and continuous pain in the left upper jaw region. Imaging exams showed the presence of a dental element, closely related to the maxillary sinus. This was removed using a conservative surgical technique.

Conclusion: The treatment of impacted teeth requires the evaluation by a trained professional to make the best decision about the conduct for each case, which can be a conservative or surgical treatment.

Key words: Molar, Third. Tooth, impacted. Maxillary sinus.

\section{NTRODUÇÃO}

A exodontia de terceiros molares superiores impactados é um procedimento cirúrgico comum. ${ }^{1}$ Essa impactação
Luís Philippe Lopes Donato ${ }^{1}$

Isabelle de Argolo Melo

Anne Carolinne Albuquerque de

Vasconcellos Costa Santos ${ }^{3}$

Lívia Maria Lopes de Oliveira ${ }^{4}$

Helder Lima Rebelo ${ }^{5}$

Hugo Franklin Lima de Oliveira ${ }^{6}$

${ }^{1}$ Cirurgião-Dentista formado pela Faculdade de Odontologia CESMAC.

E-mail: luuisphilippe@gmail.com

${ }^{2}$ Cirurgiã-Dentista formada pela Universidade

Federal de Alagoas (UFAL),

E-mail: argoloisabelle@gmail.com

${ }^{3}$ Pós-graduanda pelo ÁPICE cursos em odon-

tologia

E-mail: anne.albuquerque@hotmail.com

${ }^{4}$ Doutoranda em Odontologia pela Universidade Federal de Pernambuco (UFPE)

Universidade Federal de Pernambuco

E-mail: livialopesperiodontia@gmail.com

${ }^{5}$ Mestre em Odontologia pela Universidade

Federal de Pernambuco (UFPE),

Universidade Federal de Pernambuco

E-mail: rebelo_al@hotmail.com

${ }^{6}$ Mestre em odontologia pela Universidade

Federal de Pernambuco (UFPE)

E-mail: hugobmf@outlook.com

Telefone: (81) 99109-1005

Autor de Correspondência

Hélder Lima Rebelo. Av. Prof. Moraes Rego,

1235, Cidade Universitária - Recife - PE, Brazil,

50670-901. Email: Rebelo_al@hotmail.com.

Phone: +55 8121268817

Submetido em: 24-1-2021

Aceito: 18-3-2021 
dos dentes permanentes pode ser devido a causas locais ou sistêmicas. Os fatores locais, mais frequentemente associados, incluem a retenção prolongada de dentes decíduos, mau posicionamento dos germes dos permanentes, extensão da arcada dentária insuficiente, dente supranumerário, tumores odontogênicos, trajetória de erupção anormal e fissura labiopalatina. As causas sistêmicas mais comuns são as deficiências endócrinas, doenças febris, a Síndrome de Down e irradiação. ${ }^{1}$

Os dentes que mais comumente se tornam impactados são os terceiros molares superiores e inferiores, seguidos dos caninos superiores, pré-molares inferiores, pré-molares superiores e segundos molares superiores e inferiores. ${ }^{1,2}$ As inclusões ocorrem mais frequentemente em dentes que erupcionam numa época mais tardia. ${ }^{3}$

Os seios maxilares podem variar, em tamanho e forma, de um indivíduo para o outro, ou mesmo entre os lados direito e esquerdo do mesmo indivíduo. Em aproximadamente metade da população, seu comprimento também varia. ${ }^{4}$

Dentes que têm relação íntima com o seio maxilar precisam, durante a cirurgia, de uma melhor exposição, com movimentos de luxação cautelosos, afim de que não sejam, inadvertidamente, deslocados para essa cavidade anatômica. ${ }^{5.6}$ Porém, os deslocamentos iatrogênicos acontecem, e são responsáveis por 0,6 a 3,8\% dos casos de introdução de corpos estranhos nos seios paranasais. ${ }^{7,8}$ Elementos acidentalmente deslocados para aos seios maxilares devem ser removidos o quanto antes, para evitar sinusite.,

O objetivo desse trabalho é descrever a remoção de um terceiro molar superior esquerdo, impactado em região íntima ao seio maxilar, sem que ocorresse exposição do mesmo durante o ato operatório, diminuindo o risco de complicações trans e pós-operatórias para o paciente.

\section{RELATO DE CASO}

Paciente, gênero feminino, 29 anos, leucoderma, compareceu a clínica de cirurgia do Centro Universitário Maceió (CESMAC) localizada em Maceió-AL, com queixa principal de dor intensa e contínua, na região maxilar superior esquerda. Durante anamnese, a paciente relatou dor, inicialmente, de pouca intensidade na região, que evoluiu em um período de dois anos para uma dor mais intensa. Na história médica, foi apurado que a paciente não apresentava doença sistêmica.

Ao exame físico extra e intraoral não foram encontradas alterações clínicas, como presença de alterações de cor, volume ou fístulas. Nos exames de imagem, entretanto, foi possível observar a presença de um elemento dentário, intimamente relacionado ao seio maxilar (figuras 1, 2 e 3). Clinicamente, após análise da arcada e contagem dos dentes, foi possível avaliar que esse seria o terceiro molar superior esquerdo (28). Houve a proposição a paciente para sua remoção.

O planejamento operatório envolveu a realização de profilaxia antibiótica com Amoxicilina $1 \mathrm{~g}$, associado à Dexametasona $8 \mathrm{mg}$, uma hora antes do procedimento. Após os procedimentos de antissepsia local, foi feita anestesia por bloqueio do nervo alveolar 


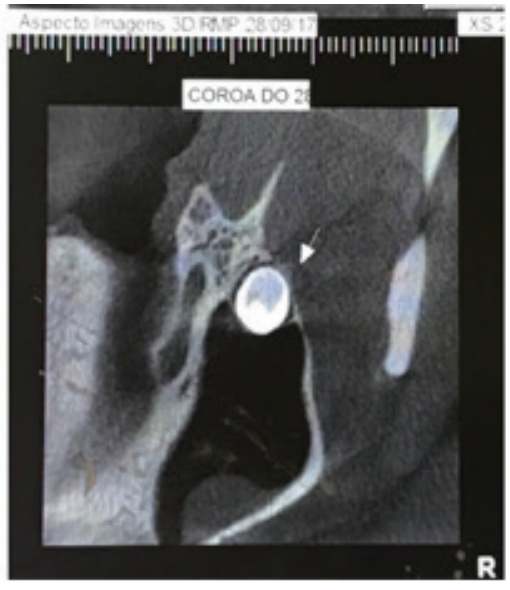

Figura 1 - Corte axial mostrando a coroa do elemento 28 em região de seio maxilar.

Fonte: Arquivo pessoal.

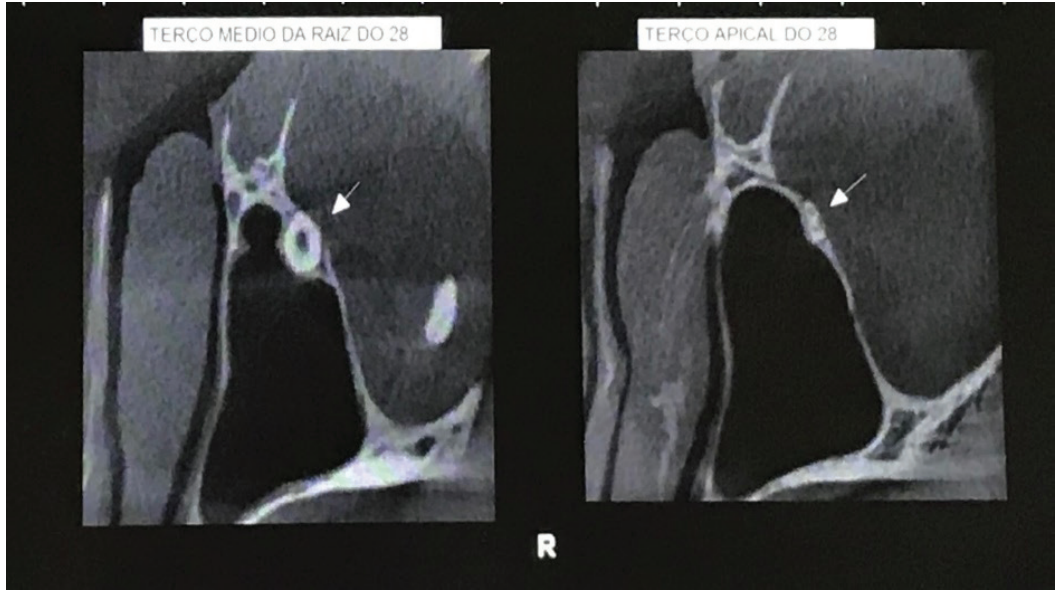

Figura 2 - Imagem tomográfica. Corte sagital dos terços médio e apical do 28.

Fonte: Arquivo pessoal.

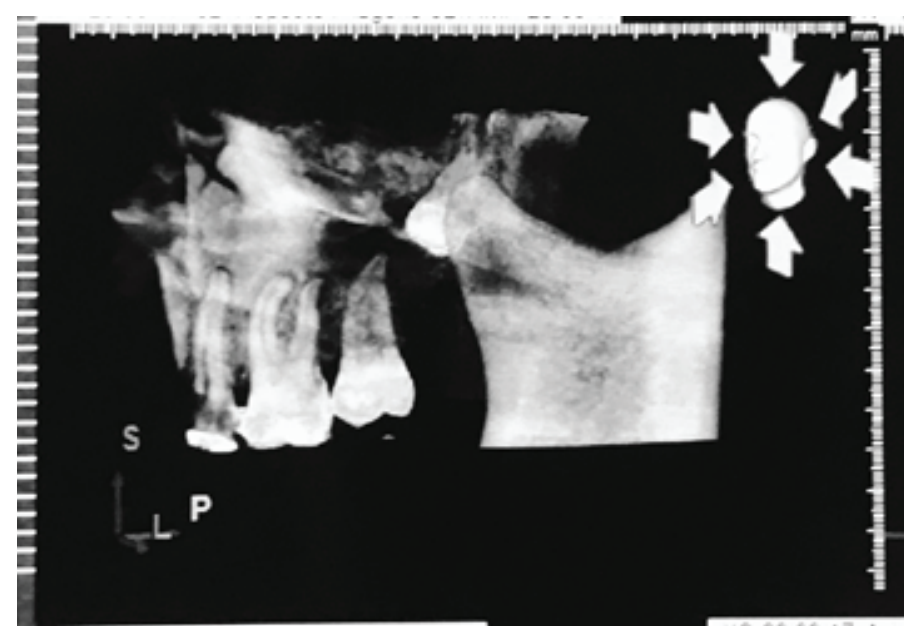

Figura 3 - Relação do elemento 28 com os dentes adjacentes. Fonte: Arquivo pessoal.

superior posterior, nervo palatino maior, complementada por anestesias infiltrativas supraperiostais, vestibular e palatina. $\mathrm{O}$ anestésico utilizado foi a lidocaína $2 \%$ com epinefrina 1:100.000 (DFL, Rio de Janeiro, Brasil). Para o caso, foi realizado um retalho triangular, envolvendo gengiva inserida, gengiva livre e papila dental com uma incisão relaxante na região distal de segundo pré-molar superior esquerdo (25).

Posteriormente, executou-se a confecção de uma janela óssea na parede maxilar, com broca esférica diamantada, sob irrigação constante com soro fisiológico. Após a osteotomia e irrigação, foi possível localizar o elemento dental e removê-lo (figura 4), com o auxílio dos elevadores de Seldin (Quinelato ${ }^{\circledR}$, São Paulo, Brasil) e de uma Pinça Hemostática Kelly (Quinelato ${ }^{\circledR}$, São Paulo, Brasil).

Figura 4- Elemento 28 após remoção. Fonte: Arquivo pessoal. 


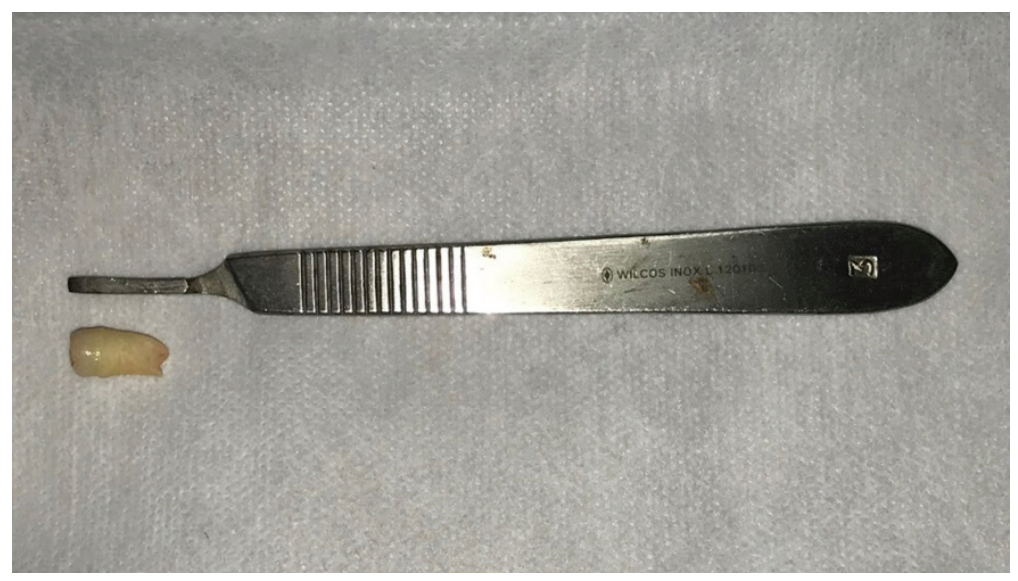

Com o retalho reposicionado, uma sutura simples foi realizada com fio de sutura Seda 4-0. Para o pós-operatório, foram feitas as prescrições de Amoxicilina + ácido clavulânico 875mg e Dipirona 500 mg, além de orientações quanto a dieta e instrução de higiene oral. Não houve complicações no pós-operatório. A sutura foi removida após sete dias, ocasião em que foi observado um padrão satisfatório de cicatrização.

\section{DISCUSS ÃO}

O paciente deve ser submetido à avaliação clínica e radiográfica periódica para detectar alterações quanto ao período de erupção dentária o mais precocemente possível, a fim de planejar e otimizar a abordagem terapêutica que deverá ser proposta ao paciente. Além disso, esse deve estar ciente das indicações, contraindicações, riscos e benefícios do manejo conservador ou remoção cirúrgica desses dentes. Dependendo da apresentação clínica do caso, a opção pelo tratamento cirúrgico pode resultar em maior risco para o deslocamento de dentes para o seio maxilar, durante o ato operatório. ${ }^{8,9}$

Dentes nessa localização podem ser a causa da produção de secreções faríngicas e nasais, vermelhidão na região da pele do lado afetado, podendo o paciente apresentar ainda sensibilidade à pressão e no vestíbulo, especialmente na região zigomática e de molares, por estar em posição ectópica e ser reconhecido pelo organismo como corpo estranho. Sensibilidade à percussão, também pode estar presente, mesmo em dentes com vitalidade, formação de abscesso vestibular ou palatino, edema difuso dos tecidos moles vestibulares, febre e dor latejante. ${ }^{10}$

No presente caso, a paciente não apresentava todos os sinais e sintomas citados anteriormente mencionados, sendo o único sintoma/queixa principal, dor na região de molares superiores com duração de dois anos. O elemento dentário estava em região íntima ao seio maxilar, sendo separado desse por uma delgada parede óssea (figuras 1, 2 e 3). O acesso cirúrgico foi planejado em função dessa localização anatômica, através de radiografias e tomografia computadorizada. A tomografia computadorizada foi escolhida em razão de sua maior precisão em relação às técnicas radiográficas convencionais ${ }^{11}$, possibilitando análise das imagens em três dimensões e volume ósseo adjacente ao dente.

A tomografia computadorizada permitiu à visualização de um aumento da densidade óssea, de formato semicircular, contínua a parede anterior do seio maxilar es- 
querdo. Imagem compatível com retenção de muco, presença de cristas ósseas alveolares conservadas, com presença dos dentes 26 e 27 além de retenção do elemento dentário 28 , que encontra-se mesioangulado (figura 2). Esse elemento estava localizado na região acima da tuberosidade da maxila, do lado esquerdo, lateralmente a fossa pterigopalatina. A coroa estava em íntimo contato com a cortical da parede posterior do seio maxilar esquerdo, com as raízes fusionadas, havendo curvatura do ápice em direção a cortical, correspondente à parede posterior do seio maxilar.

Nessas situações podem ocorrer complicações trans-operatórias, como o deslocamento dos dentes para o seio e sangramento intra-operatório do plexo venoso na área da fossa pterigoide. ${ }^{2}$ A tomografia possibilitou, portanto, um melhor planejamento desse caso, facilitando a decisão para que uma técnica cirúrgica conservadora fosse selecionada.

Os procedimentos cirúrgicos bucais, por estarem em contato com o trato alimentar, são classificados como potencialmente contaminados, sendo esperada uma taxa de infecção em torno de $10 \%{ }^{8}$ No presente relato foi realizada profilaxia antibiótica com o objetivo de evitar infecções pós-operatórias, com Amoxicilina 1g. Houve ainda a opção da realização e analgesia preemptiva com a seleção da Dexametasona $8 \mathrm{mg}$. Tanto a amoxicilina quanto a dexametasona foram administrados 1 hora antes do procedimento. $\mathrm{O}$ uso de corticosteroides com o objetivo de auxiliar a minimizar o inchaço, trismo e dor, tem ganhado ampla aceitação dos profissionais que realizam cirurgias bucomaxilofaciais, com o objetivo de aumentar o conforto do paciente durante o pós-operatório. ${ }^{1}$ Para o pós-operatório, a paciente recebeu orientações de higiene relacionadas a higiene oral, além do uso de Amoxicilina + ácido clavulânico, durante dez dias, associada ao uso de clorexidina 0,12\% (colútorio) duas vezes ao dia, 30 minutos após a escovação.

De acordo com Sigron et al ${ }^{12}$, o risco de infecção depende, em grande parte, da higiene bucal pré-operatória, do tipo de fechamento da ferida cirúrgica e do comportamento pós-operatório do paciente. Esses fatores justificaram a seleção da conduta pós-operatória para o presente caso.

\section{CONSIDERAÇÕES FINAIS}

O tratamento de dentes impactados requer a avaliação por um profissional capacitado para tomar a melhor decisão acerca da conduta para cada caso, podendo ser um tratamento conservador ou cirúrgico. São de fundamental importância, uma anamnese minuciosa associada à exames de imagens, que permitam um bom planejamento pré-operatório, capaz de evitar complicações trans e pós-operatórias.

\section{REFERÊNCIAS}

1. Miloro M, Ghali GE, Larsén PE, Waite PD. Princípios de cirurgia bucomaxilofacial de Peterson. 3. ed. São Paulo: Santos; 2016.

2. Lanzer M, Pejicic R, Kruse AL, Schneider T, Grätz KW, Lübbers HT. Anatomic (positional) variation of maxillary wisdom teeth with special regard to the maxillary sinus. Swiss Dent J 2015;125 (5): 555-571.

3. Freitas R. Tratado de cirurgia bucomaxilofacial. 1. ed. São Paulo: Santos; 2006. 
4. Torres RDG, Sotelo LRR, Vaz SLA, Bóscolo SMA, Bóscolo FN. Association between maxillary sinus pathologies and healthy teeth. Braz J Otorhinolaryngol 2016; 82(1): 33-8. https://doi.org/10.1016/j.bjorl.2015.11.004

5. Pereira Junior AJA et al. Deslocamento acidental de terceiro molar superior para o seio maxilar: relato de caso. Odontol. Clín. Cient. 2020;19(1):81-4.

6. Medeiros PJ et al. Cirurgia dos dentes inclusos. Extração e aproveitamento.1.ed. São Paulo: Santos; 2003.

7. Amorim KS, Silva VT, Cunha RS, Souto MLS, Mateus CRS, Souza LMA. Removal of an Upper Third Molar from the Maxillary Sinus. Case Reports in Dentistry 2015. https://doi.org/10.1155/2015/517149

8. Bouquet A, Coudert JL, Bourgeois D, Mazoyer JF, Bossard D. Contributions of reformatted computed tomography and panoramic radiography in the localization of third molars relative to the maxillary sinus. Oral Surgery, Oral Medicine, Oral Pathology, Oral Radiology and Endodontology 2004; 98(3): 342-347. https://doi.org/10.1016/j.tripleo.2004.02.082

9. Gil JN, Gil LF. Cirurgia do terceiro molar impactado: passo a passo. 1.ed. São Paulo: Santos; 2012.

10. Primo BT, Stringhini DJ, Costa DJ, Rebellato NLB, Scariot R. Delayed removal of maxillary third molar displaced into the maxillary sinus. Baltic Dental and Maxillofacial Journal 2016; 18: 128-32.

11. Sailer HF, Pajarola GF.Cirurgia Bucal. 1 ed. Porto Alegre: Artmed; 2000.

12. Belloti A, Costa FS, Camarini ET. Deslocamento de terceiro molar superior para o seio Maxilar: relato de caso. Rev. Cir. Traumatol. Buco-Maxilo-facial (Camaragibe) 2008; 8 (4): 35 - 40.

13. Sigron GR, Pourmand PP, Mache B, Stadilinger B, Locher MC. The most common complications after wisdom-tooth removal. Swiss Dent J 2014; 124 (10):1042-1046. 